\title{
White adipose tissue IFN- $\gamma$ expression and signalling along the progression of rodent cancer cachexia
}

\author{
Alex Shimura Yamashita ${ }^{a}$, Rodrigo Xavier das Neves ${ }^{b}$, José Cesar Rosa-Neto ${ }^{\mathrm{b}}$, Fábio dos Santos Lira ${ }^{\mathrm{c}}$, \\ Miguel Luís Batista Jr. ${ }^{\mathrm{d}}$, Paulo Sérgio Alcantara ${ }^{\mathrm{b}, \mathrm{e}}$, José Pinhata Otoch ${ }^{\mathrm{b}, \mathrm{e}}$, Marília Seelaender ${ }^{\mathrm{b}, *}$ \\ ${ }^{a}$ Department of Physiology and Biophysics, Institute of Biomedical Sciences and Faculdade de Medicina, University of Sao Paulo, São Paulo, Brazil \\ ${ }^{\mathrm{b}}$ Cancer Metabolism Research Group, Institute of Biomedical Sciences and Faculdade de Medicina, University of Sao Paulo, São Paulo, Brazil \\ ${ }^{\mathrm{c}}$ Immunometabolism Research Group, Department of Physical Education, Universidade Estadual Paulista (UNESP), Presidente Prudente, SP, Brazil \\ ${ }^{\mathrm{d}}$ Laboratory of Adipose Tissue Biology, Center for Integrated Biotechnology, University of Mogi das Cruzes, Mogi das Cruzes, São Paulo, Brazil \\ ${ }^{\mathrm{e}}$ Department of Clinical Surgery, University Hospital, University of Sao Paulo, São Paulo, Brazil
}

\section{A R T I C L E I N F O}

\section{Article history:}

Received 20 May 2015

Received in revised form 27 February 2016

Accepted 29 February 2016

Available online 14 March 2016

\section{Keywords:}

Cancer-cachexia

White adipose tissue

Interferon- $\gamma$ signalling

\begin{abstract}
A B S T R A C T
Cachexia is associated with increased morbidity and mortality in cancer. The White adipose tissue (WAT) synthesizes and releases several pro-inflammatory cytokines that play a role in cancer cachexia-related systemic inflammation. IFN- $\gamma$ is a pleiotropic cytokine that regulates several immune and metabolic functions. To assess whether IFN- $\gamma$ signalling in different WAT pads is modified along cancer-cachexia progression, we evaluated IFN- $\gamma$ receptors expression (IFNGR1 and IFNGR2) and IFN- $\gamma$ protein expression in a rodent model of cachexia $(7,10$, and 14 days after tumour implantation). IFN- $\gamma$ protein expression was heterogeneously modulated in WAT, with increases in the mesenteric pad and decreased levels in the retroperitoneal depot along cachexia progression. Ifngr1 was up-regulated 7 days after tumour cell injection in mesenteric and epididymal WAT, but the retroperitoneal depot showed reduced Ifngr1 gene expression. Ifngr 2 gene expression was increased 7 and 14 days after tumour inoculation in mesenteric WAT. The results provide evidence that changes in IFN- $\gamma$ expression and signalling may be perceived at stages preceding refractory cachexia, and therefore, might be employed as a means to assess the early stage of the syndrome.
\end{abstract}

(C) 2016 Elsevier Ltd. All rights reserved.

\section{Introduction}

Cancer-associated cachexia is a syndrome clinically characterised by alterations in energy intake, and muscle and white adipose tissue (WAT) wasting. It cannot be fully reverted by conventional nutritional support, and is markedly associated with decreased survival. Furthermore, it augments chemotherapyrelated toxicity, and presents major clinical relevance [1]. Three stages may be distinguished in this multifactorial syndrome, ranging from pre-cachexia to refractory cachexia, and effective therapies aiming at preventing the onset of the last stage are the focus of a growing number of studies [2].

The aetiology of cancer-cachexia is not completely understood and several factors are involved, including systemic inflammation, metabolic alterations and the action of tumour-related factors,

\footnotetext{
* Corresponding author at: Cancer Metabolism Research Group, Institute of Biomedical Sciences, University of São Paulo, Av. Lineu Prestes, 1524, CEP 05508900, Butantã, São Paulo, SP, Brazil.

E-mail address: seelaend@icb.usp.br (M. Seelaender).
}

such as proteolysis-inducing factor and lipid mobilizing factor/zi nc- $\alpha$-2-glycoprotein [3]. WAT inflammation is a common finding in human cachexia and in animal models [4-7]. The anatomical localisation of the adipose depot determines its relative contribution to systemic inflammation in cachexia $[4,8]$. Therefore, there is regional specificity of WAT in regard to this aspect. Inflammation in cachexia involves the enhanced production and circulation of pro-inflammatory cytokines and eicosanoids [7]. Serum concentration of pro-inflammatory cytokines, such as TNF- $\alpha$, IL- $1 \beta$, IL- 6 and IFN- $\gamma$ is often increased in patients and rodent models with cancercachexia [4,9-11]. We have shown that, both Walker-256 tumour bearing rats and cachectic colon cancer patients, the adipose tissue plays a relevant role in the establishment of chronic systemic inflammation [4-6].

As part of the adaptive immune response, IFN- $\gamma$ is produced by immune cells and acts through biding to IFN- $\gamma$ receptor subunits (IFNGR1 and IFNGR2). After binding to its receptor, intracellular signal transduction induces the target genes mediating the biological functions of this cytokine $[12,13]$. Initially identified as an antiviral effector, IFN- $\gamma$ acts upon several aspects of immune 
response and, more recently, has been shown to induce cachexia in rodent models [14-16]. However, the role of IFN- $\gamma$ in cancer cachexia progression is not fully elucidated. In this study, we examined IFN- $\gamma$ expression and signalling modification in different WAT pads along the aggravation of cachexia in the Walker-256 rodent model.

\section{Material and methods}

\subsection{Animals}

Male adult Wistar rats (200-250 g), obtained from the Institute of Biomedical Sciences, University of Sao Paulo, were maintained under a $12 \mathrm{~h}$ light $/ 12 \mathrm{~h}$ dark cycle (lights on at $6 \mathrm{~h} 00$ ), and controlled temperature conditions $\left(23 \pm 1{ }^{\circ} \mathrm{C}\right)$, receiving water and food (commercial chow, Nuvilab1, Nuvital, Brazil) ad libitum. The Ethical Committed for Animal Research (Institute of Biomedical Sciences/University of São Paulo) approved all of the adopted procedures, which were carried out in accordance with the Ethical Principles stated by the Brazilian College of Animal Experimentation; protocols n8041/2005.

\subsection{Experimental design}

To evaluate IFN- $\gamma$ expression along cancer-associated cachexia progression, rats $(n=48)$ were randomly divided into four groups ( $n=12$ /group): one group was euthanized before tumour inoculation (T0 group); in animals of the other three groups, Walker-256 tumour cells $\left(2 \times 10^{7}\right.$ cells $)$ in a $1 \mathrm{ml}$ PBS solution were injected subcutaneously, into the right flank [17]. Rats were euthanized on days 7,10 , and 14 (T7, T10 and T14, respectively) after tumour cell injection. At the end of the protocol, animals were fasted overnight ( $12 \mathrm{~h}$ ) and killed by decapitation at the start of the light cycle. Such experimental design was previously characterised and employed by our group, and shown to induce decrease in body weight, food intake, skeletal muscle and WAT wasting, in addition to reduction of adipocyte cross section area 14 days after tumour inoculation $[8,18]$. Three WAT depots, the mesenteric (MEAT), retroperitoneal (RPAT), and epididymal (EAT) pads, were excised, frozen in liquid nitrogen, and stored at $-80^{\circ} \mathrm{C}$, until analysis. All experiments were performed with whole tissue (cell populations were not isolated) fragments. Serum samples were obtained by centrifugation of blood at $1500 \mathrm{~g}$ for $10 \mathrm{~min}$ at $4{ }^{\circ} \mathrm{C}$ and then stored at $-80^{\circ} \mathrm{C}$.

\subsection{Serum determinations}

Serum total cholesterol, triglycerides (TG), low-density lipoprotein (LDL), and high density lipoprotein (HDL) were quantified using commercial kits (Labtest ${ }^{\circledR}$, Brazil). Glycerol and nonesterified fatty acid were quantified using Free Glycerol Reagent kit (Sigma, USA) and HR Series NEFA-HR (Wako, Japan), respectively.

\subsection{WAT adipokine levels}

Frozen WAT from rat samples $(0.4-0.7 \mathrm{~g})$ were homogenised in RIPA buffer (0.625\% Nonidet P-40, $0.625 \%$ sodium deoxycholate, $6.25 \mathrm{mM}$ sodium phosphate, and $1 \mathrm{mM}$ ethylene-diamine tetraacetic acid at pH 7.4), containing protease inhibitor cocktail (Sigma). Homogenates were centrifuged at $12,000 \mathrm{~g}$ for $10 \mathrm{~min}$ at $4{ }^{\circ} \mathrm{C}$, the supernatant was collected, and protein concentration was determined by Bradford assay (Bio-Rad, USA) with bovine serum albumin as reference. WAT whole tissue samples from the 3 pads were employed for the assessment of IFN- $\gamma$ protein expression by ELISA (DuoSet ELISA, R\&D Systems, USA). For IFN- $\gamma$ (DY485) assay sensitivity was found to be $5.0 \mathrm{pg} / \mathrm{ml}$ in the range of $31.2-2000 \mathrm{pg} / \mathrm{ml}$. The intra- and inter-assay variability for IFN- $\gamma$ was $2.7-5.2 \%$ and $4.9-9.5 \%$, respectively. All samples were run as duplicates, and the mean value was reported.

\subsection{Quantitative PCR}

Quantitative real-time PCR was performed as previously described [19]. In brief, frozen whole tissue samples of WAT (100-150 mg) different depots was employed to extract total RNA with the phenol-chloroform method, using the Trizol reagent (Invitrogen Life Technology, USA). The first strand of cDNA was generated from $1 \mu \mathrm{g}$ of total RNA in the presence of Oligo dT and MMLV reverse transcriptase, according to manufacturer's instruction (Invitrogen Life Technology). PCR amplification was performed in duplicate using SYBR Green ${ }^{\circledR}$ PCR Master Mix (Applied Biosystems, USA) in an ABI 7300 Real-time PCR System (Applied Biosystems) using primers as shown in Supplementary Table 1. Gene expression was normalised to the RPL19 gene. Data were calculated employing the $2^{-\Delta \Delta C T}$ method and are presented as the fold change in gene expression relative to the control sample [20].

\subsection{Statistical analysis}

Data were analysed by one-way analysis of variance (ANOVA) and expressed as means \pm SEM. Pearson correlation coefficient $(R)$ was adopted to determine the relationship between food intake and tumour weight. P-value of $<5 \%$ was considered statistically significant. The statistical analysis was performed with GraphPad Prism (Prism 5.01; GraphPad Software, La Jolla, CA).

\section{Results}

3.1. Characterisation of cancer-cachexia progression in the Walker256 rat model

As illustrated in Table 1, our previous studies showed body weight loss (range 8.9-11\%) and mesenteric WAT mass alterations in Walker-256 tumour-bearing rats, when compared with the control group $[8,18,21-23]$. In addition, several inflammatory markers (e.g. TNF- $\alpha$, IL-6, and IL-1 $\beta$ ) were upregulated in WAT in our model, as well as, tumour bearing animals presented anorexia in one study (Table 1). Supporting these data, in this study we observed a progressive reduction of food intake, a remarkeble and feature of cancer cachexia, during the 14 days following tumour cell injection (reaching $-11 \%$ on day $14 ; \mathrm{p}<0.05$ ) (Fig. $1 \mathrm{~A}$ ). As expected, tumour weight increased along cancer cachexia progression (Fig. 1B). To assess the possible relation between food intake reduction and tumour growth, we performed correlation analysis between the parameters, and no association was observed in T7 and T10. Decreased food intake was significantly correlated with tumour weight in $\mathrm{T} 14\left(R^{2}=-0.52 ; \mathrm{P}=0.01\right)$ (Fig. $\left.1 \mathrm{C}\right)$.

Table 1

Cancer cachexia features observed in Walker-256 tumour-bearing rats.

\begin{tabular}{lllllll}
\hline $\begin{array}{l}\text { Weight } \\
\text { loss }(\%)\end{array}$ & $\begin{array}{l}\text { Anorexia } \\
(\%)\end{array}$ & $\begin{array}{l}\text { TNF- } \alpha \\
(\%)\end{array}$ & $\begin{array}{l}\text { IL-6 } \\
(\%)\end{array}$ & $\begin{array}{l}\text { IL-1 } \beta \\
(\%)\end{array}$ & $\begin{array}{l}\text { Mesenteric adipose } \\
\text { tissue mass (\%) }\end{array}$ & Ref. \\
\hline-14 & ND & 39 & 38 & ND & 25 & {$[21]^{\mathrm{a}}$} \\
-8.4 & ND & ND & ND & ND & -40 & {$[8]$} \\
ND & -21 & ND & ND & ND & ND & {$[22]$} \\
-11 & ND & ND & ND & ND & 4 & {$[18]$} \\
-11 & ND & 87 & 33 & 570 & -42 & {$[23]^{\mathrm{a}}$}
\end{tabular}

a Cytokine levels in mesenteric white adipose tissue in tumour-bearing rats when compared with control group. ND: not determined. 

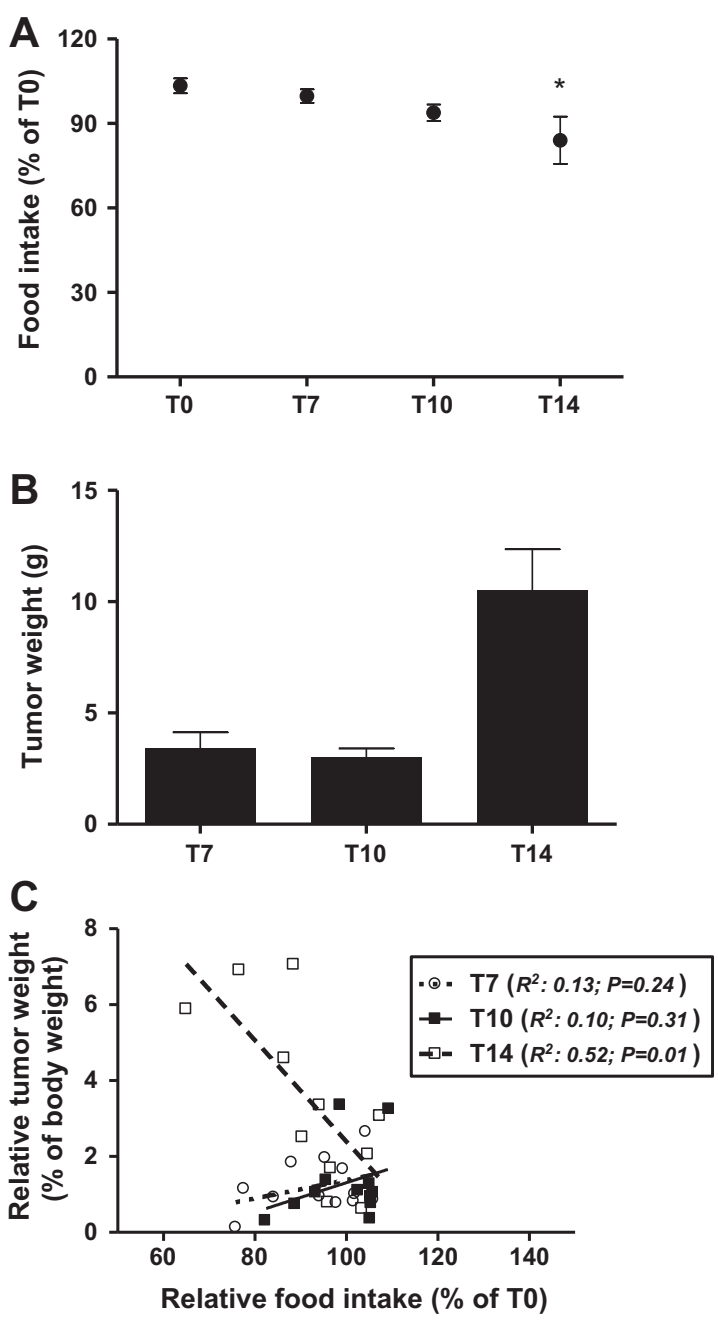

Fig. 1. Characterisation of cancer-cachexia progression. (A) Reduction in food intake along cachexia progression. Food intake was assessed at the indicated time points and expressed as percentage of day 0 consumption. Data are means \pm SEM $(\mathrm{n}=12) .{ }^{*} \mathrm{P}<0.05$ vs day 0 . (B) Absolute tumour mass. Data are means $\pm \mathrm{SEM}$ $(n=12)$. (C) Correlation between relative tumour weight and relative food intake.

\section{Table 2}

Lipid profile, glucose and protein plasma concentration in tumour-bearing rats in the different phases of cancer cachexia.

\begin{tabular}{lrrrr}
\hline & \multicolumn{1}{l}{ T0 } & \multicolumn{1}{l}{ T7 } & \multicolumn{1}{c}{ T10 } & \multicolumn{1}{c}{ T14 } \\
\hline NEFA (mE/mL) & $0.75 \pm 0.04$ & $0.80 \pm 0.04$ & $3.51 \pm 1.31$ & $1.14 \pm 0.20^{*} \dagger$ \\
Glycerol $(\mathrm{mg} / \mathrm{mL})$ & $0.14 \pm 0.04$ & $0.09 \pm 0.01$ & $0.14 \pm 0.04$ & $0.11 \pm 0.02$ \\
TAG $(\mathrm{mg} / \mathrm{mL})$ & $54.9 \pm 5.3$ & $82.8 \pm 27.0^{*}$ & $70.4 \pm 20.2$ & $89.5 \pm 15.2^{*}$ \\
Cholesterol $(\mathrm{mg} / \mathrm{mL})$ & $63.0 \pm 9.4$ & $58.4 \pm 4.7$ & $64.3 \pm 8.7$ & $62.9 \pm 5.6$ \\
LDL $(\mathrm{mg} / \mathrm{mL})$ & $16.9 \pm 2.5$ & $15.2 \pm 1.7$ & $19.0 \pm 2.6$ & $19.2 \pm 2.4$ \\
HDL $(\mathrm{mg} / \mathrm{mL})$ & $33.3 \pm 2.2$ & $31.4 \pm 2.5$ & $33.3 \pm 3.2$ & $31.3 \pm 1.1$ \\
Glucose $(\mathrm{mg} / \mathrm{mL})$ & $134.9 \pm 6.2$ & $125.3 \pm 10.5$ & $120.6 \pm 8.3^{*}$ & $113.4 \pm 12.8^{*}$ \\
Protein $(\mathrm{mg} / \mathrm{mL})$ & $43.8 \pm 4.6$ & $43.9 \pm 7.0$ & $40.2 \pm 5.4$ & $36.7 \pm 5.3^{* \dagger}$
\end{tabular}

Lipid profile, glucose and protein were measured 0 (T0), 7 (T7), 10 (T10) and 14 (T14) days after tumour implantation. Data are express as mean \pm SEM $(n=8)$. NEFA: non-esterified fatty acids; TAG: triglycerides; LDL: low-density lipoprotein; HDL: high-density lipoprotein.

${ }^{*} P<0.05$ vs T0.

${ }^{\dagger} P<0.05$ vs T7.

Table 2 shows the lipid profile, serum glucose and protein content along the progression of cachexia. Triglyceride concentration augmented 50\% $(\mathrm{P}<0.05)$ and 63\% $(\mathrm{P}<0.05)$ in T7 and T14, respectively, and NEFA increased 1.5 -fold $(\mathrm{P}<0.05)$ in T14, compared with $\mathrm{T} 0$. A progressive decrease in serum glucose concentration was observed, starting on day 10 after tumour inoculation, and the lowest concentration was observed in T14. Total protein concentration was similarly reduced in T14 (Table 2).

\subsection{INF- $\gamma$ protein expression in WAT along the progression of cachexia}

IFN- $\gamma$ alterations were examined during cachexia progression. A progressive increase of INF- $\gamma$ protein expression was observed in MEAT, reaching the peak in T14 (2.2-fold in T14 vs T0; $\mathrm{P}<0.05)$. On the other hand, in RPAT, INF- $\gamma$ protein expression decreased progressively with the lowest concentration being found in T14 $(-50 \%$ in T14 vs T0; $\mathrm{P}<0.05)$, while no alteration was detected in INF- $\gamma$ levels in EAT (Fig. 2).

\subsection{IFN- $\gamma$ receptor expression along cachexia progression}

Ifngr1 gene expression was up-regulated in MEAT (2.6-3.1-fold vs T0; $\mathrm{P}<0.05)$, reaching the peak seven days after tumour cell injection. In EAT, Ifngr1 gene expression was increased 5.3 and 3.4-fold in T10 and T14, respectively, when compared with T0 $(\mathrm{P}<0.05 ; \quad \mathrm{P}<0.05)$. A reduction of Ifngr1 gene expression (51-64\% vs T0 group; $\mathrm{P}<0.01$ ) was found four days after tumour cell injection in RPAT (Fig. 3A). Ifngr2 gene expression suffered up-regulation (2.5-fold vs T0; $\mathrm{P}<0.05$ ) in MEAT in T7 and T10 (Fig. 3B). Cachexia did not change Ifngr2 gene expression in EAT and RPAT (Fig. 3B) for any of the time points studied.

\section{Discussion}

The hallmarks of cancer cachexia are anorexia, weight loss, skeletal muscle and WAT catabolism, and systemic inflammation $[3,24]$. WAT is a key component of the pathophysiology of cachexia, as it not only suffers marked metabolic and morphological rearrangement, yet also reacts by producing many cytokines and chemoattractants, which induce local and systemic inflammation, as described for the herein adopted animal model of cachexia and for cachectic patients [25]. We have previously shown that WAT produces TNF- $\alpha$, IL- 6 , and IL-1 $\beta$, in addition to MCP-1 and other inflammatory factors in cachexia [21]. In spite of the vast knowledge on the role of cytokines, such as IL-6 and TNF- $\alpha$ in the setting of this paraneoplastic syndrome, not so much is known regarding INF- $\gamma$ signalling and receptors. To the best of our knowledge, there are no available studies showing INF production by WAT in cachexia, despite of this cytokine's potential importance in the modulation of inflammation. Recently, we demonstrated that the production of TNF- $\alpha$, IL-1 $\beta$, IL- 6 and IL-10 in WAT of Walker 256 tumour-bearing rats is dependent on time and on

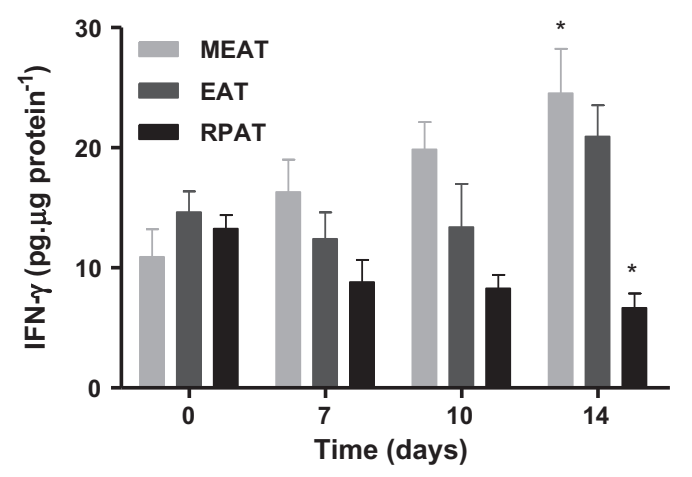

Fig. 2. Depot-specific modulation in IFN- $\gamma$ protein expression along experimental cachexia progression in WAT. Data are expressed as means \pm SEM $(n=8)$. MEAT: mesenteric adipose tissue; RPAT: retroperitoneal adipose tissue; EAT: epididymal adipose tissue. ${ }^{*} P<0.05$ vs day 0 . 

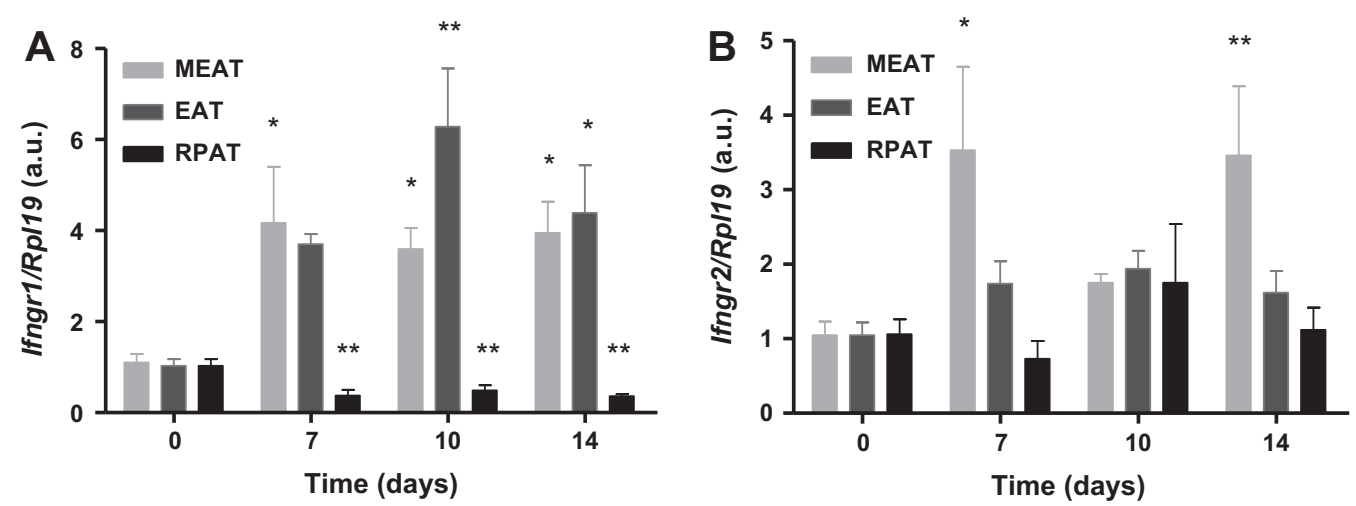

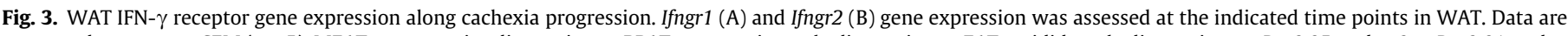

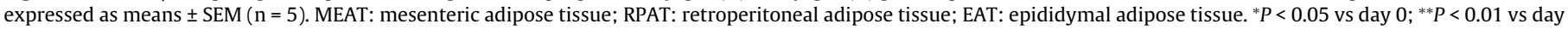
0.

the anatomical localisation of the pad [23]. These results were shown to be related with heterogeneous activation of the inflammasome and of the NFKB pathways in adipocytes, and in whole adipose tissue samples. We now report that, in the same cachexia model, WAT-associated IFN- $\gamma$ content and receptor expression likewise present a time-dependent and depot-specific alteration.

Downstream effectors of IFN- $\gamma$ signalling are JAK-STAT pathway and more recently described, the IRF transcription factor family members [26]. For instance, IRF4 transcription factor sensitive isoproterenol-induced lipolysis in vitro and in vivo and upregulates the transcription of key lipid metabolism-associated genes, which could control, at least partly, lipid catabolism in WAT [27]. Interestingly, adipocytes isolated from cachectic cancer patients are more sensitive to noradrenaline-induced lipolysis [28]. However, whether IFN- $\gamma$ sensitizes cells to noradrenaline-induced lipolysis or acts simply in a synergic manner in the process, remains to be further evaluated. The present data clearly indicate that IFN- $\gamma$ pathway is upregulated in a depot-specific and timedependent form in rat WAT, suggesting that IFN- $\gamma$ may be important for lipid metabolism regulation and WAT wasting modulation in cachexia.

IFN- $\gamma$ may contribute to WAT depletion by controlling the transcription of several enzymes involved in lipid metabolism [29]. IFN- $\gamma$ reduces LPL activity in WAT and lipid uptake in 3T3-L1 cells [30]. In addition, corroborating this hypothesis, in adipocytes or in treated mice, IFN- $\gamma$ robustly augments NEFA content [31]. A second potential mechanism for WAT depletion during cachexia is the induction of adipose cell death. However, 3T3-L1 cells treated with IFN- $\gamma$ do not present enhanced apoptosis, suggesting specific modulation of metabolism function rather than cytotoxic effects as a mechanism of IFN- $\gamma$ action [32]. The physiological outcome of systemic IFN- $\gamma$ increase is insulin resistance and the increase of NEFA, through reduction of glucose uptake and enhanced lipolysis in adipocytes and likely, in skeletal muscle cells, all of which are frequently observed in cachexia patients [33]. In the present study, IFN- $\gamma$ could be strongly associated with WAT lipolysis, as we found higher levels of FFA in late-stage cachexia (Table 2), in parallel with the progressive increase of IFN $-\gamma$ protein expression in WAT, along the time course protocol. This finding is interesting, as it suggests that adipose biopsies (preferentially of subcutaneous adipose tissue in patients) might predict the depth of the cachectic stage. A limitation of the study is that we did not assess which adipose tissue cell population was associated with the changes. Since immune cells produce IFN $-\gamma$ and both tumour-bearing rats and cachectic patients show marked macrophage infiltration in adipose pads $[7,12]$, it is at this time, impossible to rule out a major contribution of such cells or any other component of the stromal- vascular fraction. Nevertheless, we may argue that for clinical purposes, whole tissue measurements present higher interest.

WAT depot-specific cytokine modulation in cachexia has been previously shown and with the present data we add information regarding this aspect [6,34]. The increased protein expression of IFN- $\gamma$ in WAT observed along cancer cachexia may contribute to the twofold increase in serum IFN- $\gamma$ of Walker-256 tumourbearing rats [16]. In addition, it is worth to note that serum levels of IFN- $\gamma$ are augmented in advanced prostate carcinoma [11]. Immunocompromised mice inoculated with IFN- $\gamma$ producing tumour cells or, still, rats treated with repetitive doses of recombinant murine IFN- $\gamma$ develop anorexia-cachexia in few days [14,15]. On the other hand, IFN- $\gamma$ monoclonal antibody administration attenuates weight loss in these rodent models and increases survival by $50 \%$ in tumour-bearing animals, when compared with controls $[14,15]$.

In conclusion, we present evidence that IFN- $\gamma$ signalling pathway is in a time and depot-specific manner dysregulated in the WAT of cachectic tumour-bearing rats, which could contribute, as already shown for other adipose tissue-derived cytokines, for cachexia-associated systemic inflammation.

\section{Conflict of interest}

The authors declare that there is no conflict of interest.

\section{Authors' contributions}

ASY, RXN, JCR, FSL, MLB, PSA and JPO and MS designed the study, ASY, RXN, JCR and FSL performed the experiments, and ASY, RXN, JCR, FSL, MLB and MS analysed the data. ASY, RXN and MS wrote the paper.

\section{Funding}

Funding for this study was provided by FAPESP Grant 2012/50079-0 (Marília C.L. Seelaender).

\section{Acknowledgement}

We thank Emilia Ribeiro for her technical contribution.

\section{Appendix A. Supplementary material}

Supplementary data associated with this article can be found, in the online version, at http://dx.doi.org/10.1016/j.cyto.2016.02.015. 


\section{References}

[1] P.J. Ross et al., Do patients with weight loss have a worse outcome when undergoing chemotherapy for lung cancers?, Br J. Cancer 90 (10) (2004) 19051911.

[2] K. Fearon et al., Definition and classification of cancer cachexia: an international consensus, Lancet Oncol. 12 (5) (2011) 489-495.

[3] M.J. Tisdale, Mechanisms of cancer cachexia, Physiol. Rev. 89(2)(2009)381-410.

[4] M.L. Batista Jr. et al., Adipose tissue-derived factors as potential biomarkers in cachectic cancer patients, Cytokine 61 (2) (2013) 532-539.

[5] R.G. Camargo et al., NF-kappaBp65 and expression of its pro-inflammatory target genes are upregulated in the subcutaneous adipose tissue of cachectic cancer patients, Nutrients 7 (6) (2015) 4465-4479.

[6] F.S. Lira et al., Exercise training decreases adipose tissue inflammation in cachectic rats, Horm. Metab. Res. 44 (2) (2012) 91-98.

[7] A.P. Machado, L.F. Costa Rosa, M.C. Seelaender, Adipose tissue in Walker 256 tumour-induced cachexia: possible association between decreased leptin concentration and mononuclear cell infiltration, Cell Tissue Res. 318 (3) (2004) 503-514.

[8] M.L. Batista Jr. et al., Heterogeneous time-dependent response of adipose tissue during the development of cancer cachexia, J. Endocrinol. 215 (3) (2012) 363-373.

[9] R. Talar-Wojnarowska et al., Tumor necrosis factor alpha and interferon gamma genes polymorphisms and serum levels in pancreatic adenocarcinoma, Neoplasma 56 (1) (2009) 56-62.

[10] P. Ravasco, I. Monteiro-Grillo, M. Camilo, How relevant are cytokines in colorectal cancer wasting?, Cancer J 13 (6) (2007) 392-398.

[11] E. Tazaki et al., Serum cytokine profiles in patients with prostate carcinoma, Exp. Ther. Med. 2 (5) (2011) 887-891.

[12] K. Schroder et al., Interferon-gamma: an overview of signals, mechanisms and functions, J. Leukoc. Biol. 75 (2) (2004) 163-189.

[13] B. Saha et al., Gene modulation and immunoregulatory roles of interferon gamma, Cytokine 50 (1) (2010) 1-14.

[14] P. Matthys et al., Severe cachexia in mice inoculated with interferon-gammaproducing tumor cells, Int. J. Cancer 49 (1) (1991) 77-82.

[15] H.N. Langstein et al., The roles of gamma-interferon and tumor necrosis factor alpha in an experimental rat model of cancer cachexia, Cancer Res. 51 (9) (1991) 2302-2306.

[16] A. Folador et al., Effect of fish oil supplementation for two generations on changes of lymphocyte function induced by Walker 256 cancer cachexia in rats, Nutr. Cancer 61 (5) (2009) 670-679.

[17] F.S. Lira et al., Effect of endurance training upon lipid metabolism in the liver of cachectic tumour-bearing rats, Cell Biochem. Funct. 26 (6) (2008) 701-708.
[18] M. Beluzi et al., Pioglitazone treatment increases survival and prevents body weight loss in tumor-bearing animals: possible anti-cachectic effect, PLoS ONE 10 (3) (2015) e0122660

[19] A.S. Yamashita et al., Notch pathway is activated by MAPK signaling and influences papillary thyroid cancer proliferation, Transl. Oncol. 6 (2) (2013) 197-205.

[20] M.W. Pfaffl, A new mathematical model for relative quantification in real-time RT-PCR, Nucl. Acids Res. 29 (9) (2001) e45.

[21] F.F. Donatto et al., Resistance exercise modulates lipid plasma profile and cytokine content in the adipose tissue of tumour-bearing rats, Cytokine 61 (2) (2013) 426-432.

[22] F.S. Lira et al., Hypothalamic inflammation is reversed by endurance training in anorectic-cachectic rats, Nutr. Metab. (Lond) 8 (1) (2011) 60

[23] R.X. Neves et al., White adipose tissue cells and the progression of cachexia: inflammatory pathways, J. Cachexia Sarcopenia Muscle (2015), http://dx.doi. org/10.1002/jcsm.12041.

[24] A. Laviano et al., Neural control of the anorexia-cachexia syndrome, Am. J Physiol. Endocrinol. Metab. 295 (5) (2008) E1000-E1008.

[25] M. Seelaender et al., Inflammation in cancer cachexia: to resolve or not to resolve (is that the question?), Clin. Nutr. 31 (4) (2012) 562-566.

[26] K. Ozato, P. Tailor, T. Kubota, The interferon regulatory factor family in host defense: mechanism of action, J. Biol. Chem. 282 (28) (2007) 20065-20069.

[27] J. Eguchi et al., Transcriptional control of adipose lipid handling by IRF4, Cell Metab. 13 (3) (2011) 249-259.

[28] T. Agustsson et al., Mechanism of increased lipolysis in cancer cachexia, Cancer Res. 67 (11) (2007) 5531-5537.

[29] R.A. Memon et al., In vivo effects of interferon-alpha and interferon-gamma on lipolysis and ketogenesis, Endocrinology 131 (4) (1992) 1695-1702.

[30] J.S. Patton et al., Interferons and tumor necrosis factors have similar catabolic effects on 3T3 L1 cells, Proc. Natl. Acad. Sci. USA 83 (21) (1986) 8313-8317.

[31] W. Khazen et al., Acute and selective inhibition of adipocyte glyceroneogenesis and cytosolic phosphoenolpyruvate carboxykinase by interferon gamma, Endocrinology 148 (8) (2007) 4007-4014.

[32] F.C. McGillicuddy et al., Interferon gamma attenuates insulin signaling, lipid storage, and differentiation in human adipocytes via activation of the JAK/ STAT pathway, J. Biol. Chem. 284 (46) (2009) 31936-31944.

[33] M.A. Honors, K.P. Kinzig, The role of insulin resistance in the development of muscle wasting during cancer cachexia, J. Cachexia Sarcopenia Muscle 3 (1) (2012) 5-11.

[34] A.S. Yamashita et al., Depot-specific modulation of adipokine levels in rat adipose tissue by diet-induced obesity: the effect of aerobic training and energy restriction, Cytokine 52 (3) (2010) 168-174. 